A

1896

\title{
LIST OF THE BIRDS
}

\section{EASTERN NORTH AMERICA}

FOR SAI.E BY

SMITHSONIAN BRADLEY WHIDDEN

CHARLES B. CORY

DEC 041987

\section{LMBRARIES}

BOSTON

1896 

LIST OF THE BIRDS

\section{EASTERN NORTH AMERICA}

FOR SALE BY

SMITHSONIAN BRADLEY WHIDDEN

DEC $04198 \%$ 18 ARCH St., Boston

\section{LIBRARIES}

CHARLES B. CORY

$$
\begin{gathered}
\text { BOSTON } \\
1896
\end{gathered}
$$



Cory, Charles Barney

List of the birds of eastern North America

1896

Birds

PREFACE.

IN the following list special reference is made to species which occur in New England, Illinois, and Florida. Taking these States to form the points of an imaginary triangle, a somewhat crude idea of the range of species which occur within this area may be obtained, as it is evident that birds which occur in Illinois, New England, and Florida must, in all probability, also occur in most of the intermediate States; whereas those which are recorded from only one of these localities would probably be species which only touched within the limits of this area during their migrations, or birds of comparatively local distribution. Of course, a number of the species do not occur in either New England, Illinois, or Florida, as the list includes all birds which are known to occur in North America east of the ninetieth meridian.

CHARLES B. CORY. 



\section{A LIST}

OF THE

\section{BIRDS OF EASTERN NORTII AMERICA.}

FAMILY PODICIPID E. Grebes.

A. O. 0.

(2) COL MBUS HOLBELLII (REINH.).

Hulbœll's Grebe.

N. E. IIl.

2 (3) COLYMBUS AURITUS LINN.

Horned Grebe.

N. E. Fla. Ill.

3 (4) COLYMBUS NIGRICOLLIS CALIFORNICUS (HEERM.).

American Eared Grebe.

III.

Ranges from Mississippi Valley Westward. Does not occur in the Atlantic States. (Illinois Ridgway.)

L 4 (6) PODILYMBUS PODICEPS (LiNN.).

Pied-billed Grebe.

N. E. Fla. Ill.

FAMILY URINATORIDE. LOONS.

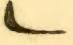

5 (7) URINATOR IMBER (GUNN.).

Loon.

N. E. Fla. Ill.

6 (9) URINATOR ARCTICUS (LINN.).

Black-throated Loon.

N. E. IIl.?

L 7 (11) URINATOR LUMME (GUNN.).

Red-throated Loon.

N. E. Fla. 111.

FAMILY ALCIDÆ. Auks, Murres, and Puffins.

8 (12) LUNDA CIRRHATA PALL.

Tufted Puffin.

Pacific species claimed by Audubon to have once been taken on coast of Maine. No other record.

9 (13) FRATERCULA ARCTICA (LINN.).

Puffin.

N. E.

10 (13 $a$ ) FRATERCULA ARCTICA GLACIALIS (TEMM.).

Large-billed Puffin.

N. E. 
A. $0, v$.

11 (27) CEPPHUS GRYLLE (LINN.).

Black Guillemot.

N. E.

12 (28) CEPPHUS MANDTII (Licht.).

Mandt's Guillemot.

N. E.

13 (30) URIA TROILE (LINN.).

Murre.

N. E.

14 (31) URIA LOMVIA (LINN.).

Brünnich's Murre.

N. E.

15 (32) ALCA TORDA LiNN.

Razor-billed Auk.

N. E.

16 (33) PLAUTUS IMPENNIS (LINN.).

Great Auk.

N. E.

Extinct species.

17 (34) ALLE ALLE (LinN.).

Dovekie.

Little Auk.

N. E.

FAMILY STERCORARIIDAE. Skuas and Jaegers.

18 (35) MEGALESTRIS SKUA (BRUNN.).

Skua.

N. E. IIl.

L

19 (36) STERCORARIUS POMARINUS (TEMM.).

Pomarine Jaeger.

N. E.

20 (37) STERCORARIUS PARASITICUS (LINN.).

Parasitic Jaeger.

N. E. Fla. $I 11$.

21 (38) STERCORARIUS LONGICAUDUS VIEILL.

Long-tailed Jaeger.

N. E. Fla. Ill.

FAMILY LARIDIAE. Gulls and Terns.

22 (39) GAvia ALBA (GUNn.).

Ivory Gull.

Rather rare winter visitant to New Brunswick, and probably northern New England.

23 (40) RISSA TRIDACTYLA (LINN.).

Kittiwake Gull.

N. E. III.

24 (42) LARUS GLAUCUS BRÜNN.

Glaucous Gull.

N. E. III.

25 (43) LARUS LEUCOPTERUS FABER.

Iceland Gull.

N. E. III. 
A. $0.8 \dot{~}$
No.

26 (45) LARUS KUMLIENI BREWST.

Kumlien's Gull.

N. E.

27 (47) LARUS MARINUS LINN.

Great Black-backed Gull.

N. E. Fla. Ill.

28 (50) LARUS AFFINIS REINH.

Siberian Gull.

Old World species recorded from Greenland.

29 (51) LARUS ARGENTATUS BRÜNN.

Herring Gull.

III.

European species, accidental on the Atlantic coast of N. A.

30 (51a) LARUS ARGENTATUS SMITHSONIANUS COUES.

American Herring Gull.

N. E. Fla. Ill.

31 (54) LARUS DELAWARENSIS ORD.

Ring-billed Gull.

N. E. Fla. IIl.

32 (56) LARUS CANUS LINN.

Mew Gull.

European species recorded from Labrador (one record).

33 (58) LARUS ATRICILLA LINN.

Laughing Gull.

N. E. Fla. IIl.?

34 (59) LARUS FRANKLINII Sw. \& RICH.

Franklin's Gull.

III.

Not common east of the Mississippi River; not found on the Atlantic coast.

35 (60) LARUS PHILADELPHIA (ORD.).

Bonaparte's Gull.

N. E. Fla. Ill.

36 (60.1) LARUS MINUTUS PALL.

Little Gull.

European species; only once taken on our coast.

37 (61) RHODOSTETHIA ROSEA (MACGIL.).

Ross's Gull.

Northern species, recorded from Alaska and Greenland.

38 (62) XEMA SABINII (SAB.).

Sabine's Gull.

N. E. IIl.

39 (63) GELOCHELIDON NILOTICA (HASSELQ.).

Gull-billed Tern.

N. E. Fla. Ill.

40 (64) STERNA TSCHEGRAVA LEPECH.

Caspian Tern.

N. E. Fla. IIl.

41 (65) STERNA MAXIMA BODD.

Royal Tern.

N. E. Fla. 111. 
A. $0 . \mathrm{U}$.
No.

$42(67)$

STERNA SANDVICENSIS ACUFLAVIDA (CABOT).

Cabot's Tern.

N. E. Fla.

43 (68) STERNA TRUDEAUI AUD.

Trudeau's Tern.

South American species; stragglers have been taken on the Atlantic coast of United States.

44 (69) STERNA FORSTERI NUTT.

Forster's Tern.

N. E. Fla. 111 .

C. 45 (70) STERNA HIRUNDO LINN.

Common Tern.

N. E. Fla. Ill.

46 (71) STERNA PARADISÆA BRÜNN.

Arctic Tern.

N. E.

47 (72) StERna DOUgalli Montag.

Roseate Tern.

N. E. Fla.

148 (74) STERNA ANTILLARUM (LESS.),

Least Tern.

N. E. Fla. 111 .

49 (75) STERNA FULIGINOSA GMEL.

Sooty Tern.

N. E. Fla.

50 (76) STERNA AN

Bridled Tern.

Tropical species, accidental on Florida coast. South Carolina, one record.

51 (77) HYDROCHELIDON NIGRA SURINAMENSIS (GMEL.)

Black Tern.

N. E. Fla. III.

52 (78). HYDROCHELIDON LEUCOPTERA (MEISN. \& SCHINZ).

White-winged Black Tern.

Iuropean species, of accidental occurrence in the United States. Wisconsin, one record.

53 (79) ANOUS STOLIDUS (LINN.).

Noddy.

Fla.

FAMILY RHYNCHOPID瓜. Skimmers.

54 (80) RYNCHOPS NIGRA LINN.

Black Skimmer.

N. E. Fla.

\section{FAMILY DIOMEDEIDA. Albatrosses.}

55 (81) DIOMEDEA EXULANS (LINN.).

Wandering Albatross.

Species common to southern seas, claimed to have been seen at Tampa Bay, Florida. 
A. O. v.
NO.

56 (83) THALASSOGERON CULMINATUS (GOULD).

Yellow-nosed Albatross.

South Pacific species, accidental in eastern North America. St. Lawrence River, one record.

\section{FAMILY PROCELLARIIDAE. Fulmars and} Shearwaters.

57 (S6) FULMARUS GLACIALIS (LINN.).

Fulmar.

N. E.

อ̊ ( $86 a$ ) FULMARUS GLACIALIS MINOR KJJERBGLLING.

Lesser Fulmar.

N. E.

59 (88) PUFFINUS BOREALIS CORY.

Cory's Shearwater.

N. E.

60 (89) PUFFINUS MAJOR FABER.

Greater Shearwater.

N. E. Flı.

61 (90) PUFFINUS PUFFINUS (BRÜNx.).

Manx Shearwater.

N. E.

European species, accidental on the Atlantic coast.

62 (92) PUFFINUS AUDUBONI Finscr.

Audubon's Shearwater.

Fla.

63 (94) PUFFINUS STRICKLANDI RIDGW.

Sooty Shearwater.

N. E.

64 (9S) ESTRELATA HASITATA (IIUHL).

Black-capped Petrel.

Fla.

65 (99) ESTRELATA SCALARIS BREWST.

Scaled Petrel.

Rare straggler; one specimen known, taken in western New York.

66 (101) BULWERIA BULWERI (JARD. \& SELBY).

Bulwer's Petrel.

Old world species, accidental in Greenland.

67 (101) PROCELLARIA PELAGICA LiNN.

Stormy Petrel.

N. E.

(is (106) OCEANODROMA LEUCORHOA (VIEILL.).

Leach's Petrel.

N. E.

69 (109) OCEANITES OCEANICUS (KUIL).

Wilson's Petrel.

N. E. Fla.

70 (110) CYMODROMA GRALLARIA (VIEILL.).

White-bellied Petrel.

Tropical species, onco taken near St. Marks, Florida. 
A. o. v.

71 (111) PELAGODROMA MARINA (LATH.).

White-faced Petrel.

South Atlantic species, accidental off coast of Massachusetts.

FAMILY PHÄ̈THONTIDÆ. Tropic Birds.

72 (112) PHÄ̈THON FLAVIROSTRIS BRANDT.

Yellow-billed Tropic Bird.

Fla.

73 (113) PHÄ̈THON \#THEREUS LINN.

Red-billed Tropic Bird.

Accidental on Nerffoundland Banks.

FAMILY SULIDAE. Gannets.

74 (11.1) SULA CYANOPS SUND.

Blue-faced Booby.

Fla.

Accidental on Florida coast.

75 (115) SULA SULA (LINN.).

Booby.

N. E. Fla.

76 (116) SULA PISCATOR (LINN.).

Red-footed Booby.

Fla.

Accidental on Florida coast.

77 (117) SULA BASSANA (LINN.).

Gannet.

N. E. Fla.

\section{FAMILY ANHINGIDFE. Darters.}

78 (118) ANHINGA ANHINGA (LINN.).

Anhinga.

Fla. I11.

FAMILY PHALACROCORACIDEE. Cormorants.

79 (119) PHALACROCORAX CARBO (LINN.).

Cormorant.

N. E.

L 80 (120) PHALACROCORAX DILOPHUS (SW. \& RiCH.).

Double-crested Cormorant.

N. E. Fla. Ill.

81 (120a) PHALACROCORAX DILOPHUS FLORIDANUS (AUD.). Florida Cormorant.

Fla. IIl.

32. (121) PHALACROCORAX MEXICANUS (BRANDT).

Mexican Cormorant.

Western species, casual in Mississippi Valley to Illinois. 
FAMILY PELECANID AE. Pelicans.

1 A. ‥

83 (125) PELECANUS ERYTHRORHYNCHOS GMEL.

American White Pelican. N. E. Fla. IIl.

\$4 (126) PELECANUS FUSCUS LINN.

Brown Pelican.

N. E. Fla. III.

FAMILY FREGATIDAE. Man-o'-War Birds.

85 (128) FREGATA AQUILA (IINN.).

Man-o'-War Bird.

N. E. Fla.

FAMILY ANATID E. Ducks, Geese, and Swans. 86 (129) MERGANSER AMERICANUS (CASS.).

American Merganser.

Sheldrake.

N. E. Fla. 111 .

S7 (130) MERGANSER SERRATOR (LINN.).

Red-breasted Merganser.

Sheldrake.

N. E. Fla. Ill.

1 - 88 (131) LOPHODYTES CUCULlatus (LiNN.).

Hooded Merganser.

N. E. Fla. Ill.

89 (132) ANAS BOSCHAS LINN.

Mallard Duck.

N. E. Fla. Ill.

90 (133) ANAS OBSCURA GMEL。

- anas vbscura rubifhes

N. E. Fla. Ill.

91 (134) ANAS FULVIGULA RIDGW.

Florida Duck.

Florida Black Duck.

Fla.

L 92 (135) ANAS STREPERA IINN.

Gadwall.

N. E. Fla. Ill.

$\angle 93$ (136) ANAS PENELOPE LINN.

Widgeon.

European Widgeon.

N. E. III.

European species, of ten taken on our coast.

91 (137) ANAS AMERICANA GMEL.

American Widgeon. Baldpate. N. E. Fla. Ill.

- European Teal.

N. E.

Accidental in Eastern North America. New England, several records. 
A. . . . .

96 (139) ANAS CAROLINENSIS GMEL.

Green-winged Teal.

N. E. Fla. IIl.

97 (140) ANAS DISCORS LINN.

Blue-winged Teal.

N. E. Fla. I1l.

L- 98 (141) ANAS CYANOPTERA VIEILL.

Cinnamon Teal.

Fla. Ill.

L. 99 (142) SPATULA CLYPEATA (LINN.).

Shoveller Duck.

N. E. TIa. IIl.

100 (143) DAFILA ACUTA (LINN.).

Pintail Duck.

N. E. Fla. Ill.

(ـ 101 (141) AIX SPONSA (LINN.).

Wood Duck.

N. E. Fla. IIl.

102 (145) NETTA RUFINA (PALL.).

Rufous-crested Duck.

European species. New York, one record.

« 103 (146) AYTHYA AMERICANA (ETT.).

Redhead Duck.

N. E. Fla. Ill.

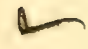

104 (147) AYTHYA VALLISNERIA (IVILS.).

Canvas-back Duck.

N. E. Fla. 111 .

105 (148) AYTHYA MARILA NEARCTICA STEJN.

American Scaup Duck.

Blue Bill or Black Head.

N. E. Fla. Ill.

106 (149) AYTHYA AFFINIS (EYT.).

Lesser Scaup Duck.

Blue Bill or Black Head. N. E. Fla. Ill.

4. 107 (150) AYTHYA COLLARIS (DONOV.).

Ring-necked Duck. N. E. Fla. Ill.

4- 108 (151) GLAUCIONETTA CLANGULA AMERICANA (BONAP.). American Golden-eye Duck.

Whistler.

N. E. Fla. 111.

Q 109 (152) GLAUCIONETTA ISLANDICA (GMLL.).

Barrow's Golden-eye Duck. N. E. Ill.

Q 110 (153) CHARITONETTA ALBEOLA (LiNN.).

Buffle-head Duck.

Dipper.

N. E. Fla.

111 (151) CLANGULA HYEMALIS (LINN.).

Old-squaw.

Long-tailed Duck.

N. E. Fla. I11. 
112 (15, HISTRIONICUS HISTRIONICUS (LINY.)

Harlequin Duck.

N. E. Fla.? IIl.

113 (1.̆li) CAMPTOLAIMUS LABRADORIUS (GMEL.).

Labrador Duck.

N. E.

Probably extinct.

114 (157) ENICONETTA STELLERI (PALL.).

Steller's Duck.

Arctic species which occurs in Greenland.

115 (159) SOMATERIA MOLLISSIMA BOREALIS (BREHM).

Northern Eider Duck.

N. E.

L 116 (160) SOMATERIA DRESSERI SIIARPE.

American Eider Duck. I. F. III.

$1117(16 \%)$ SOMATERIA SPECTABILIS (LINN.).

King Eider Duck. N. E. Ill.

L 118 (163) OIDEMIA AMERICANA Sw. \& RICH.

American Scoter Duck.

Velvet Scoter. Butter-bill Coot. N. E. Fla. Ill.

119 (161) OIDEMIA FUSCA (LINN.).

European species, recorded from Greenland.

Q 120 (165) OIDEMIA DEGLANDI BONAP.

White-winged Scoter Duck.

White-winged coot.

N. E. Fla. Ill.

1:1 (166) OIDEMIA PERSPICILLATA (LINN.).

Surf Scoter Duck.

Skunk-head Coot.

N. I. Fla. Ill.

C_.2. (167) ERISMATURA RUBIDA (WILS.).

Ruddy Duck.

N. E. Fla. 111.

123 (168) NOMONYX DOMINICUS (LINN.).

Masked Duck.

N. E.

Tropical species, accidental in eastern United States.

CHEN HYPERBOREA (PALL.).

Lesser Snow Goose.

N. E. 111 .

Rare in eastern United States.

1... (169a) CHEN HYPERBOREA NIVALIS (FOIST.).

Greater Snow Goose. N. L. Fla. Ill.

126 (169.1) CHEN CERULESCENS (LINN.). 


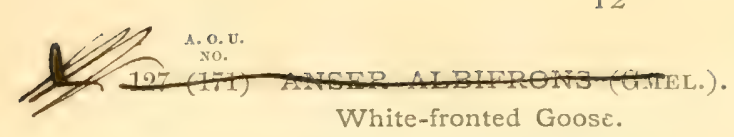

European species, recorded from Greenland.

12S (171:) ANSER ALBIFRONS GAMBELI (INATL.).

American White-fronted Goose. N. E. Ill.

1 L 129 (172) BRANTA CANADENSIS (LINN.).

Canada Goose.

N. E. Fla. I11.

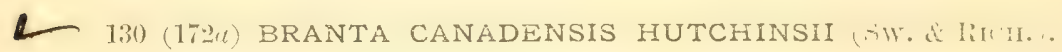
Hutchins's Goose. N. I.

L 131 (1720) BRANTA CANADENSIS MINIMA RIDGW.

Cackling Goose.

IIl.

Not recorded farther east than Illinois,

132 (173) BRANTA BERNICLA (LINN.).

Brant.

N. E. Fla. Ill.

133 (174) BRANTA NIGRICANS (LAWR.).

Black Brant.

N. F.

Western species, occasionally taken on Atlantic coast.

134 (175) BRANTA LEUCOPSIS (BECHST.).

Barnacle Goose.

N. E.

Greenland; rare on Atlantic coast of United States.

135 (179) OLOR CYGNUS (LINN.).

Whooping Swan.

Old world species which occurs in Greenland, but not elsewhere in North America.

136 (180) OLOR COLUMBIANUS (ORD.).

Whistling Swan.

N. E. Fla. IIl.

L-137 (181)

OLOR BUCCINATOR (RICH.).

Trumpeter Swan.

Western species, rare on Atlantic coast of United States. Illinois.

FAMILY PHCENICOPTERIDE. Flamingoes.

138 (182) PHENICOPTERUS RUBER (LINN.).

American Flamingo.

Fla.

FAMILY PLATALEIDFE: Spoonbills.

139 (1S3) AJAJA AJAJA (IJINN.).

Roseate Spoonbill.

Fla. 


\section{FAMILY IBIDIDE. Ibises.}

A. o. . .
No.

140 (184) GUARA ALBA (LINN.).

White Ibis.

N. E. Fla. Ill.

141 (185) GUARA RUBRA (LINN.).

Scarlet Ibis.

A tropical species; no evidence of its recent occurrence in North America.

142 (186) PLEGAdis AUTUMNALIS (HASSELQ.).

Glossy Ibis.

N. E. Fla. Ill.

143 (18i) PLEGAdIS GUARAUNA (LIMN.).

White-faced Glossy Ibis.

Western species, once recorded from Florida.

FAMILY CICONIID Fi. Storks and Wood Ibises.

144 (188) TANTALUS LOCULATOR LINN.

Wood Ibis.

Fla. Ill.

FAIVILY ARDEIDAE. Herons, Bitterns, etc.

145 (190) BOTAURUS LENTIGINOSUS (MONTAG.).

American Bittern.

N. E. Fla. 111.

146 (191) ARDETTA EXILIS (GMEL.).

Least Bittern.

N. E. Fla. IIl.

147 (191.1) ARDETTA NEOXENA CORY.

Cory's Least Bittern.

Fla.

148 (192) ARDEA OCCIDENTALIS AUD.

Great White Heron.

Fla.

Accidental in Illinois.

149 ARDEA WUERDEMANNI BAIRD.

Wuerdemann's Heron.

Fla.

Probably a colored phase of A. occidentalis, or an abnormally colored specimen of Ardea wardi.

150 (193) ARDEA WARDI RIDGW.

Ward's Heron.

Fla.

151 (194) ARDEA HERODIAS LINN.

Great Blue Heron.

N. E. Fla. III.

152 (195) ARDEA CINEREA LINX.

European Blue Heron.

Euronean species, recorded from Greenland.

153 (196) ARDEA EGRETTA GMEL.

American Egret.

N. E. Fla. I1l. 
$2154(197)$ ARDEA CANDIDISSIMA GMEL.

Snowy Heron.

N. E. Fla. III.

L 155 (198) ARDEA RUFESCENS GMEI.

Reddish Egret. Fla. Ill.

L 156 (199) ARDEA TRICOLOR RUFICOLLIS (GOSSE).

Louisiana Heron. Fla.

L- 157 (200) ARDEA CCERULEA LINN.

Little Blue Heron. N. E. Fla. IIl.

$15 \mathrm{~S}$ (201) ARDEA VIRESCENS LINA.

Green Heron. N. E. Fla. 111.

159 (202) NYCTICORAX NYCTICORAX NAEVIUS (BODD.).

Black-crowned Night Heron. N. E. Fla. Ill.

160 (203) NYCTICORAX VIOLACEUS (LINN.).

Yellow-crowned Night Heron. N. E. Fla. Ill.

FAMILY GRUIDE. Cranes.

Q 161 (201) GRUS AMERICANA (LINN.).

Whooping Crane. N. E. Fla. Ill.

162 (205) GRUS CANADENSIS (LINN.).

Little Brown Crane.

Western species, two records east of Mississippi River.

[ـ 163 (206) GRUS MEXICANA (MïLL.).

Sandhill Crane.

N. H. Fla. Ill.

\section{FAMILY ARAMID正. Courlans.}

161 (207) ARAMUS GIGANTEUS (BONAP.).

Limpkin.

Fla.

FAMILY RALLIDAE. Rails, Gallinules, and Coots.

L 105 (208) RALLUS ELEGANS Ard。

King Rail.

N. E. Fla. IIl.

166 (211) RALLUS CREPITANS GMEL.

Clapper Rail.

N. E. Fla.

167 (211a) RALLUS CREPITANS SATURATUS (RIDGW.)

Louisiana Clapper Rail.

106 (2116) RALLUS SCOTTII (SENN.).
Scott's Rail.

Fla. 
1 A. . . .

169 (212) RALLUS VIRGINIANUS LINN.

Virginia Rail.

N. E. Fla. Inl.

170 (213) PORZANA PORZANA (LINN.).

Spotted Crake.

European species, recorded from Greenland.

L 171 (214) PORZANA CAROLINA (LINN.).

Sora. Carolina Rail. N. E. Fla. Ill.

1 L 172 (215) PORZANA NOVEBORACENSIS (GMEL.).

Yellow Rail. N. E. Fla. Ill.

L 173 (216) PORZANA JAMAICENSIS (GMEL.).

Black Rail.

N. E. Fla. Ill.

174 (217) CREX CREX (LINN.).

Corn Crake.

European species, accidental in eastern North America.

L 175 (21S) IONORNIS MARTINICA (LINA.).

Purple Gallinule. N. E. Fla. Ill.

L 176 (219) GALlinula Galeata (Licht.).

Florida Gallinule.

N. E. Fla. Ill.

177 [는)

European Coot.

European species, recorded from Greenland.

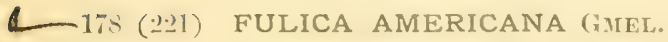

American Coot.

N. E. Fla. Ill.

FAMILY PHALAROPODIDE. Phalaropes.

L 179 (222) CRYMOPHILUS FULICARIUS (LINN.).

Red Phalarope.

N. E. Fla. Ill.

$\angle 180$ (223) PHALAROPUS LOBATUS (IINN.).

Northern Phalarope. N. E. Ill.

181 (224) PHALAROPUS TRICOLOR (VIEILI.).

Wilson's Phalarope. N. F. III.

FAMILY RECURVIROSTRIDAE. AvOces: and Stilts.

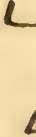

182 (225) RECURVIROSTRA AMERICANA GMEL.

American Avocet. $\quad$ X. li. Flit. Ill.

I83 (226) HIMANTOPUS MEXICANUS (MÜLL.).

Black-necked Stilt.

Y. T. Fla. Ill. 
FAMILY SCOLOPACID E. Snipes, Sandpipers, etc. A. O. .
No.

184 [227] SCOLOPAX RUSTICOLA LINN.

European Woodcock.

European species, accidental in eastern North America; several records.

185 (328) PHILOHELA MINOR (GMEL.).

American Woodcock.

N. E. Fla. Ill.

186 [229] GALLINAGo GALLINAGo (LiNN.).

European Snipe.

European species, occurs in Greenland.

187 (230) GALLINAGO DELICATA (ORD.).

Wilson's Snipe.

Jack Snipe.

N. E. Fla, 111 .

L 188 (231) MACRORHAMPHUS GRISEUS (GMEL.).

Dowitcher.

Red-breasted Snipe. N. E. Fla. Ill.

L 189 (232) MACRORHAMPHUS SCOLOPACEUS (SAT.).

Long-billed Dowitcher.

Western Red-breasted Snipe. N. E. Fla. IIl.

L 190 (233) MICROPALAMA HIMANTOPUS (BONAP.).

Stilt Sandpiper. N.E. Fla. Ill.

L 191 (234) TRINGA CANUTUS LINN.

Knot.

N. E. Fla. Ill.

192 (235) TRINGA MARITIMA BIÜNN.

Purple Sandpiper.

N. E. Fla. Ill.

- 193 (239) TRINGA MACULATA VIEILL.

Pectoral Sandpiper.

Grass-bird.

N. E. Fla. Ill.

L 194 (240) TRINGA FUSCICOLLIS VIEILI.

White-rumped Sandpiper.

Bonaparte's Sandpiper.

N. E. Fla. III.

1195 (241) TRINGA BAIRDII (COUES).

Baird's Sandpiper.

N. E. III.

Q 196 (242) TRINGA MiNuTILLA VIEILL.

Least Sandpiper.

N. E. Fla. Ill.

197 [213] TRINGA ALPINA LINN.

Dunlin.

European species, accidental in North America. 
A. $0, \mathcal{U}$

198 (243a) TRINGA ALPINA PACIFICA (COUES).

Red-backed Sandpiper.

American Dunlin. N. E. Fla. Ill.

199 (244) TRINGA FERRUGINEA BRÜNN.

Curlew Sandpiper. N. E.

European species, of rare occurrence in eastern North America.

200 (24i) EREUNETES PUSILLUS (LINN.).

Semipalmated Sandpiper. N. E. Fla. Ill.

201 (24i) EREUNETES OCCIDENTALIS LAWR.

Western Sandpiper. N. E. Fla. Ill.

$2(1) \cdot 2$ (24) CALIDRIS ARENARIA (IINT.).

Sanderling Sandpiper.

Bull Peep. N. E. Fla. Ill.

203 (249) LIMOSA FEDOA (LINN.).

Marbled Godwit.

N. E. Fla. Ill.

204 (ำ1) LIMOSA H EMMASTICA (LINN.).

Hudsonian Godwit.

Goose Bird.

N. E. Fla. Ill.

20): [2:2] LIMOSA LIMOSA (LINN.).

Black-tailed Godwit.

European species, which occurs in Greenland.

206 [253] TOTANUS NEBULARIUS (GUNy.).

Green-shank.

Old world species, accidental in Florida. No recent records.

207 (254) TOTANUS MELANOLEUCUS (GMEL.).

Greater Yellow-legs.

Winter Yellow-legs. N. E. Fla. III

208 (25.) TOTANUS FLAVIPES (GMEL.).

Yellow-legs.

Summer Yellow-legs. N. E. Fla. Ill.

$2209(2,0 i)$ TOTANUS SOLITARIUS (WILS.).

Solitary Sandpiper. N. E. Fla. Ill.

210 [25] TOTANUS OCHROPUS (LNN.).

Green Sandpiper.

European species, once recorded from Nova Scotia.

211 (258) SYMPHEMIA SEMIPALMATA (GMEL.).

Willet. N. E. Fla. Ill.

212 (25Sa) SYMPHEMIA SEMIPALMATA INORNATA BREWST.

Western Willet.

Fla, 
A. o. . .
No.

213 [260] PAVONCELlA PUGNAX (LINN.).

Ruff.

N. E.

European species, accidental in United States.

214 (261) BARTRAMIA LONGICAUDA (BECHST.).

Bartramian Sandpiper.

Upland Plover.

N. E. Fla. I11.

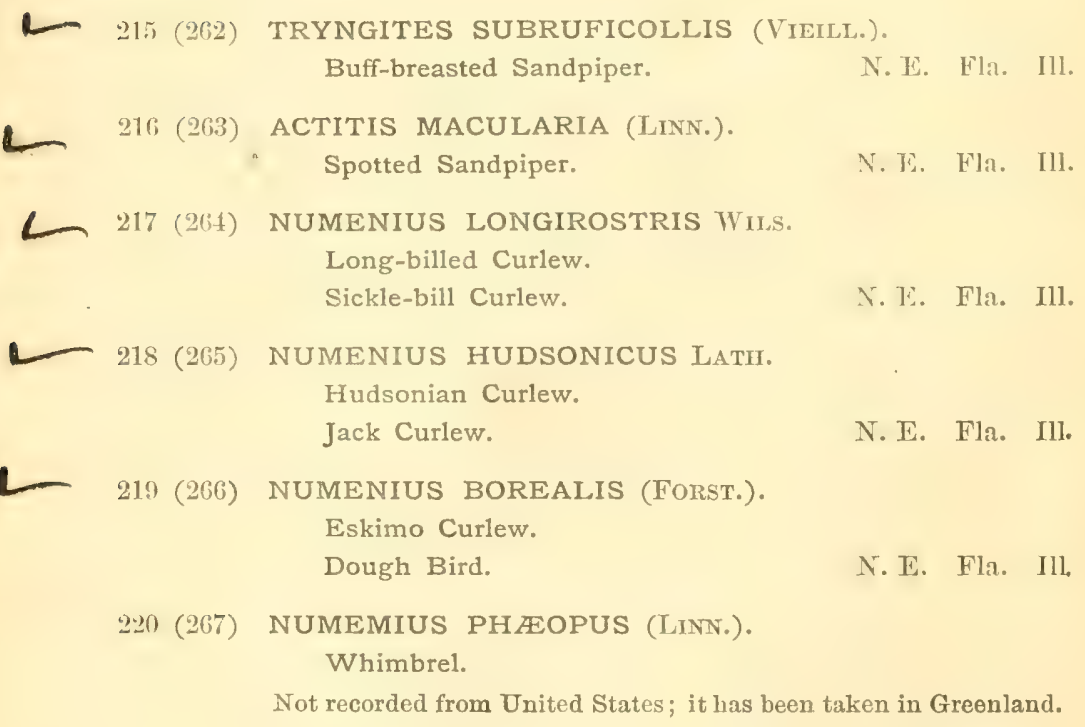

FAMILY CHARADRIIDAE. Plovers.

221 (269) VANELLUS VANELLUS (LINN.).

Lapwing.

European species, occurs in Greenland; one record for eastern United States.

22.2 (270) CHARADRIUS SQUATAROLA (LINN.).

Black-bellied Plover.

Beetle Head.

N. E. Fla. :11.

223 (271) CHARADRIUS APRICARIUS LINN.

Golden Plover.

European species, which occurs in Greenland.

2.21 (27:2) CHARADRIUS DOMINICUS MÜLI,

American Golden Plover.

Green Plover.

N. F. Fla. III.

225 (273) AEGIALITIS VOCIFERA (LINN.).

Killdeer Plover.

N. E. Fla. 111 . 
226 (274) EGIALITIS SEMIPALMATA BONAP.

Semipalmated Plover.

N. E. Fla. I11.

227 (275) AGIALITIS HIATICULA (LINN.).

Ring Plover.

Not recorded from United States; it occurs in Greenland.

228 (27\%) EGIALITIS MELODA (ORD).

Piping Plover. N. E. Fla.

229 (27\%(

Belted Piping Plover.

N. E. H1h.

230 (280) \#GIALITIS WILSONIA (OID).

Wilson's Plover.

N. E. Fla.

2:1 (2S1) ÆEGIALITIS MONTANA (TOWNS.),

Mountain Plover.

Western species, aecidental in Florida.

FAMILY APHRIZID必. Surf Birds and Turnstones. L232 (283) ARENARIA INTERPRES (LINY.).

Turnstone.

N. E. Fla. Ill.

\section{FAMILY HAEMATOPODIDE. Oyster Catchers.}

233 (285) HÆMATOPUS OSTRALEGUS LINN.

Oyster-catcher.

European species, which occurs in Greenland.

$23 \pm$ (286) HæMATOPUS PALLIATUS 'I'EMM.

American Oyster-catcher.

N. E. Fla.

FAMILY TETRAONIDAE. Grouse, Partridges, etc.

235 (289) COLINUS VIRGINIANUS (IMNN.).

Quail. Bob-white.

N. E. Fla. III.

L.23i (2R) COLINUS VIRGINIANUS FLORIDANUS (('OLES).

Florida Quail. Florida Bob-white. Fla.

237 (298) DENDRAGOPUS CANADENSIS (LINN.).

Canada Grouse. Spruce Partridge.

L_ 238 (300) BONASA UMBELLUS (LINN.).

Ruffed Grouse.

N. E. Ill.

239 (300a) BONASA UMBELLUS TOGATA (LINN.).

Canadian Ruffed Grouse. 


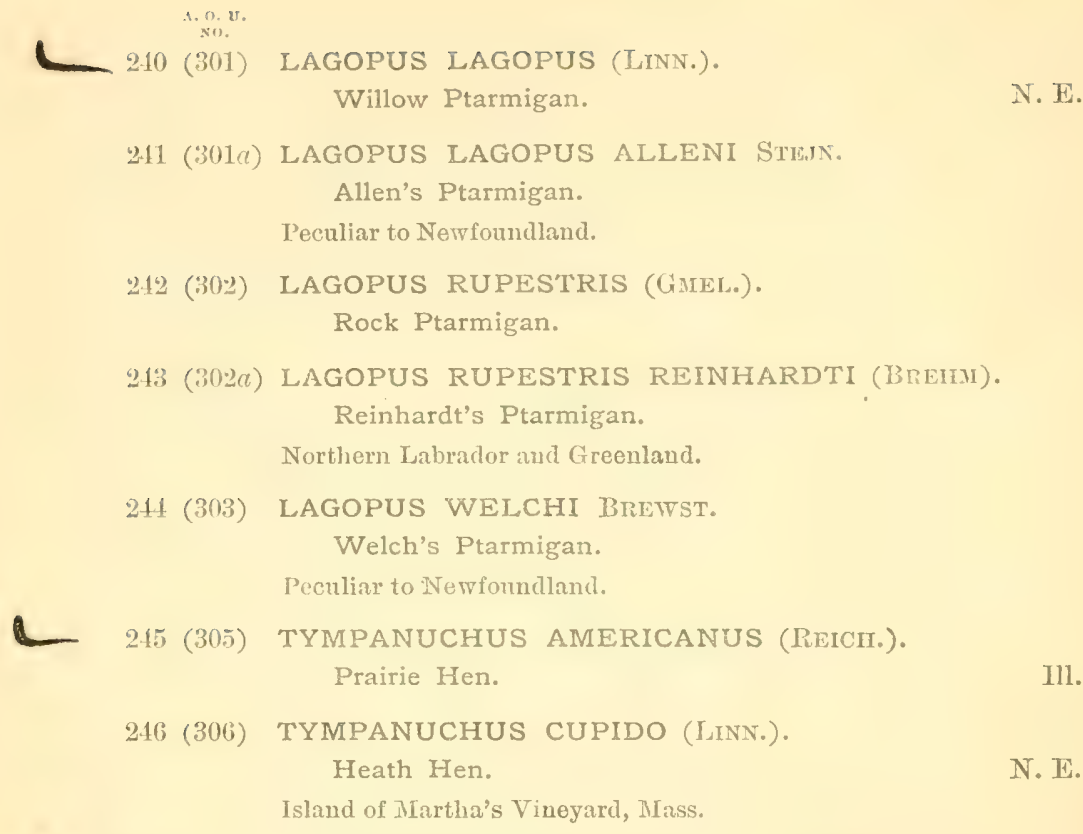

247 (30S6) PEDIOCAETES PHASIANELLUS CAMPESTRIS

Prairie Sharp-tailed Grouse. Ill. Western species, which sometimes occurs in Wisconsin and Illinois.

FAMILY PHASIANIDZE. Pheasants.

248 (310) MELEAGRIS GALLOPAVO LINN.

Wild Turkey. Northern Florida. Fla. Ill.

249 (310a) MELEAGRIS GALLOPAVO OSCEOLA SCOTT.

Florida Turkey. Southern Florida.

FAMILY COLUMBIDEC. Pigeons.

2.5) (31!) COLUMBA LEUCOCEPHALA LINN.

White-crowned Pigeon.

Fla.

$2 \pi$ (:3iT) ECTOPISTES MIGRATORIUS (LINN.).

Passenger Pigeon.

N. F. III.

M.: ("1;, ZENAIDURA MACROURA (TINN.).

Mourning Dove.

N. E. Fla. III.

2.i: (.,.) ZENAIDA ZENAIDA (BONAP.).

Zenaida Dove.

Fla.

Florida Keys. 
A. O. E.

254 (319) MELOPELIA LEUCOPTERA (LINN.).

White-winged Dove.

Fla.

Westorn species, accidental at Key West, Fla.

255 (320) COLUMBIGALLINA PASSERINA TERRESTRIS

Ground Dove.

Cirapman.

256 (322) GEOTRYGON MARTINICA (GIIEL.).

Fla.

Key West Quail-Dove.

Fla.

West Indian species, recorded from Florida Keys.

257 (32.1) GEOTRYGON MONTANA (LINN.).

Mountain Dove.

Fla.

West Indian species, once recorded from Key West, Fla.

$25 S$ (32:3) STARNCENAS CYANOCEPHALA (LINN.).

Blue-headed Quail Dove.

Cuban species, accidental on Florida Keys.

FAMILY CATHARTIDEE. American Vultures.

259 (325) CATHARTES AURA (LINN.).

Turkey Vulture. Turkey Buzzard. N. Ti. Fla. Ill.

260 (326) CATHARISTA ATRATA (BARTR.). Unuhy

Black Vulture. Carrion Crow. N. T. Fla. Ill.

FAMILY FALCOIVIDEE. Vultures, Fa!cons, Hawls, Eagles, etc.

a_ 201 (327) ELANOIDES FORFICATUS (LINN.).

Swallow-tailed Kite.

N. F. Fla. Ill.

L_262 (328) ELANUS LEUCURUS (MIEIL.).

White-tailed Kite.

Fla. III.

ᄂ 263 (329) ICTINIA MISSISSIPPIENSIS (WILS.).

Mississippi Kite.

Fla. III.

264 (330) ROSTRHAMUS SOCIABILIS (VIEI.L.).

Everglade Kite.

Fia.

2 265 (331) CIRCUS HUDSONIUS (IINN.).

Marsh Hawk.

N. E. Fla. III.

C 266 (332) ACCIPITER VELOX (Wr.s.).

Sharp-shinned Hawk.

N. F. Fla. 111.

4267 (333) ACCIPITER COOPERI (BONAP.).

Cooper's Hawk.

N. E. Flit. Ill. 
A. O.
No.

268 (334) ACCIPITER ATRICAPILLUS (IVIS.).

American Goshawk.

N. F. Fla. 111.

269 (335) BUTEO UNICINCTUS HARRISI (AUD.).

Harris's Hawk.

Western species, which occurs in Louisiana.

270 (336) BUTEO BUTEO (LinN.).

European Buzzard.

European species, once taken in United States; Michigan, one record.

(337) BUTEO BOREALIS (GMEL.).

Red-tailed Hawk.

N. E. Flat. III.

$272(337 a)$ BUTEO BOREALIS KRIDERII IOOPES.

Krider's Hawk.

I11.

273 (3376) BUTEO BOREALIS CALURUS (CASS.).

Western Red-tail.

Western species, recorded from Illinois.

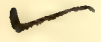

$274(338)$

BUTEO BOREALIS HARLANI (AUD.).

Harlan's Hawk.

IIl.

275 (339) BUTEO LINEATUS (GMEL.).

Red-shouldered Hawk.

N. E. Flit. Ill.

276 (339a) BUTEO LINEATUS ALLENI RIDGW.

Florida Red-shouldered Hawk.

Fla.

L- 277 (342) BUTEO SWAINSONI BONAP,

Swainson's Hawk.

N. E. II1.

Western species, occasionally occurring in eastern United States; casual in Florida.

C $27 \times$ (343) BUTEO LATISSimus (Wir.s.).

Broad-winged Hawk.

N. E. Fla. Ill.

279 (344) BUTEO BRACHYURUS VIEILL.

Short-tailed Hawk.

Fla.

Tropical species, resident in Florida.

280 (346) Asturina PLAgiata SCHLEgEL.

Mexican Goshawk.

IIl.

Western species, once observed in Illinois; no other record.

$2 S 1$ (:3iil) ARCHIBUTEO LAGOPUS SANCTI-JOHANNIS (GMLL.).

American Rough-legged Hawk.

N. E. 111.

282 (348) ARCHIBUTEO FERRUGINEUS (LiCHT.).

Ferruginous Rough-leg.

IIl.

283 (349) AQUILA CHRYSAËTOS (LINN.).

Golden Eagle.

พ. E. Fla. 111 . 
284 (351) HALIAEETUS ALBICILLA (LrNN.).

Gray Sea Eagle.

Occurs in Greenland, but has not been taken in the United States.

$\mathcal{L}_{285(352)}$

HALIFETUS LEUCOCEPHALUS (LINN.).

Bald Eagle. N. E. Fla. Ill.

286 (353) FALCO ISLANDUS BRÜNN.

White Gyrfalcon.

N. E.

287 (354) FALCO RUSTICOLUS LiNN.

Gray Gyrfalcon.

N. E.?

288 (354a) FALCO RUSTICOLUS GYRFALCO (LINN.). Gyrfalcon.

N. E.

289 (354b) FALCO RUSTICOLUS OBSOLETUS (GMEL.). Black Gyrfalcon.

N. E.

290 (355) FALCO MEXICANUS SCHLEG.

Prairie Falcon.

III.

291 (356) FALCO PEREGRINUS ANATUM (BONAP.).

Duck Hawk.

Peregrine Falcon. N. E. Fla. Ill.

L 292 (357) FALCO COLUMBARIUS LINN.

Pigeon Hawk.

N. E. Fla. Ill.

293 (358.1) FALCO REGULUS PALL.

Merlin.

European species, recorded from Greenland.

294 (359.1) FALCO TINNUNCULUS LINN.

Kestrel.

N. E.

European species, once taken in Massachusetts.

295 (360) FALCO SPARVERIUS LINN.

American Sparrow Hawk.

N. E. Fla. Ill.

296 (361) FALCO SPARVERIOIDES VIG.

Cuban Sparrow Hawk.

Fla.

297 (362) POLYBORUS CHERIWAY (JACQ.).

Audubon's Caracara.

Fila.

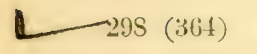

PANDION HALIAËTUS CAROLINENSIS ((IMEL.).

American Osprey. Fish Hawk. N. E. Fli. III.

FAMILY STRIGIDE. Barn Owls.

299 (365) STRIX PRATINCOLA BONAP.

American Barn Owl.

N. E. Fla. Ill. 
FAMILY BUBONIDA. Horned Owls.

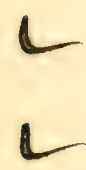
A. o. $\mathrm{v}$.
No.

300 (366) ASIO WILSONIANUS (LESS.).

American Long-eared Owl.

N. E. III.

301 (367) ASIO ACCIPITRINUS (PALL.).

Short-eared Owl.

N. E. IIl.

302 (368) SYRNIUM NEBULOSUM (FORST.).

Barred Owl.

N. E. Fla. Ill.

303 ( $368 a$ ) SYRNIUM NEBULOSUM ALLENI RIDGW.

Florida Barred Owl.

Fla.

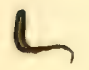

$30+(370)$ SCOTIAPTEX CINEREA (GMEL.).

Great Gray Owl. Cinereus Owl.

N. E. Ill.

Q 305 (3\%1) NYCTALA TENGMALMI RICHARDSONI (BONAP.).

Richardson's Owl.

N. E. I1l.

$1-306$ (372) NYCTALA ACADICA (GMEL.).

Saw-whet Owl. Acadian Owl.

N. E. 111 .

L 307 (373) MEGASCOPS ASIO (LINN.).

Screech Owl.

N. E. III.

308 (373a) MEGASCOPS ASIO FLORIDANUS (RIDGW.).

Florida Screech Owl.

Fla.

L 309 (3\%) BUBO VIRGINIANUS (GMEL.).

Great Horned Owl.

N. E. Fla. IIl.

310 (375a) BUBO VIRGINIANUS SUBARCTICUS (HOY).

Western Horned Owl.

III.

Western race; specimens have been taken in Illinois and $W$ isconsin.

311 (375c) BUBO VIRGINIANUS SATURATUS RIDGW.

Dusky Horned Owl.

Labrador and Hudson's Bay, westward.

L- 312 (376) NYCTEA NYCTEA (LiNN.).

Snowy Owl.

N. E. Ill.

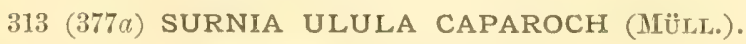

American Hawk Owl.

N. E. III.

314 (378) SPEOTYTO CUNICULARIA HYPOGEA (BONAP.).

Burrowing Owl.

N. E.

Western species, one record from Massachusetts.

315 (378 $\alpha$ ) SPEOTYTO CUNICULARIA FLORIDANA RIDGW.

Florida Burrowing Owl.

Fla. 
A. 0. v.
No.

FAMILY PSITTACIDA.

316 (382) CONURUS CAROLINENSIS (LINN.).

Carolina Paroquet.

Fla.

FAMILY CUCULIDA. Cuckoos, Anis; etc.

317 (383) CROTOPHAGA ANI LINN.

Ani.

West Indian species, recorded from Florida, Louisiana, and Ponnsylvania.

318 (386) COCCYZUS MINOR (GIIEL.).

Mangrove Cuckoo.

Fla.

West Indian species, casual in Southern Florida.

319 (386a) COCCYZUS MINOR MAYNARDI RIDGW.

Maynard's Cuckoo.

Fla.

Southern Florida.

$3: 0$ (3S7) COCCYZUS AMERICANUS (LINN.).

Yellow-billed Cuckoo. N. E. Fla. Ill.

L 321 (388) COCCYZUS ERYTHROPHTHALMUS (WILS.).

Black-billed Cuckoo.

N. E. Fla. III.

FAMILY ALCEDINIDAE. Kingfishers.

1 322 (390) CERYLE ALCYON (LINN.).

Belted Kingfisher.

X. E. Fla. Ill.

FAMILY PICID死. Woodpeckers.

32:? (392) CAMPEPHILUS PRINCIPALIS (LINN.).

Ivory-billed Woodpecker.

Fla. Ill.

324 (393) DRYOBATES VILLOSUS (LINN.).

Hairy Woodpecker.

N. E. Ill.

325 (3936) DRYOBATES VILLOSUS AUDUBONII (SWAINS.).

Southern Hairy Woodpecker.

Fla.

326 (391) DRYoBATES PUBESCENS Mftlíanus

Downy Woodpecker.

N. E. Flat. I11.

327 (395) DRYOBATES BOREALIS (VIEILL.).

Red-cockaded Woodpecker.

Fla.

1 - 328 (400) PICOIDES ARCTICUS (SWAINS.).

Arctic Three-toed Woodpecker.

N. E. III. 
A. o. v.
No.

329 (101) PICOIDES AMERICANUS BREHM.

American Three-toed Woodpecker.

N. E.

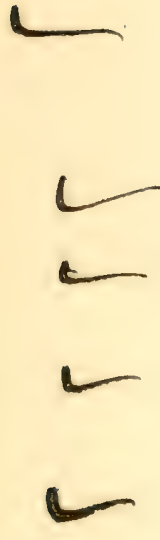

330 (402) SPHYRAPICUS VARIUS (LINN.).

Yellow-bellied Sapsucker.

Yellow-bellied Woodpecker. \& N.T. Fla. Ill.

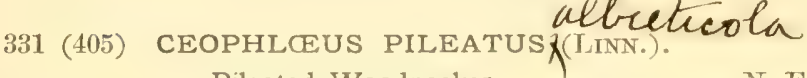

Pileated Woodpecker.

N. E. Fla. Ill.

332 (114) MELANERPES ERYTHROCEPHALUS

Red-headed Woodpecker.

(IINN.).

N. E. Fla. 111.

333 (409) MELANERPES CAROLINUS (LINN.).

Red-bellied Woodpecker.

N. E. Flan Ill.

334 (412) COLAPTES AURATUS (LINN.).

Flicker.

Golden-winged Woodpecker.

N. E. Fla. Ill.

FAMILY CAPRIMULGIDAE. Goatsuckers.

335 (416) ANTROSTOMUS CAROLINENSIS (GMEL.).

Chuck-will's-widow.

N. E. Fla. 111 .

336 (417) ANTROSTOMUS VOCIFERUS (WILS.).

Whip-poor-will.

N. E. Fla. 111.

— 337 (420) CHORDEILES VIRGINIANUS (GMEL.).

Nighthawk. $\quad$ N. E. Fla. 111.

7 2 338 (420 $)$ CHORDEILES VIRGINIANUS HENRYI ( (isS.).

merecend

Western Nighthawk.

IIl.

Western species, recorded from Illinois.

339 (420c) CHORDEILES VIRGINIANUS CHAP:IANI (MENETT).

Florida Nighthawk.

Fla.

FAMILY MICROPODID $Æ$. Swifts.

340 (423) CHAETURA PELAGICA (LINN.).

Chimney Swift.

N. E. Fla. 111.

FAMILY TROCHILID EE. Hummingbirds.

311 (12S) TROCHILUS COLUBRIS IINN.

Ruby-throated Hummingbird.

N. F. Fla. 111. 


\section{FAMILY TYRANNID}

A. $0, \mathrm{U}$.

342 (412) MILVULUS TYRANNUS (IINN.).

Fork-tailed Flycatcher.

South American species, occasionally taken in the United States; recorded from New Jersey aud Mississippi.

343 (443) MILVUlus FORFICATUS (GHel.).

Scissor-tailed Flycatcher.

N. E. Fla.

Western species, accidental in eastem United States.

TYRANNUS TYRANNUS (LINN.).

Kingbird.

N. E. Fla. Ill.

345 (445) TYRANNUS DOMINICENSIS (GMEL.).

Gray Kingbird.

N. E. Fla.

Semi-tropical species, not uncommon in Florida; accidental in Georgia and South Carolina.

346 (447) TYRANNUS VERTICALIS SAY.

Arkansas Kingbird.

N. E.

Western species, accidental east of the Mississippi; one record for New England.

$L$
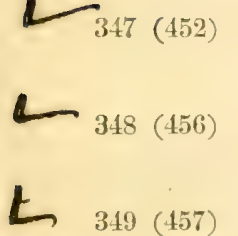

348

$(456)$

349

$(457)$

)

MYIARCHUS CRINITUS (LINN.).

Crested Flycatcher.

N. E. Fla. Ill.

SAYORNIS PHCEBE (IATIY.).

Phœbe.

N. E. Fla. III.

SAYORNIS SAYA (BONAP.).

Say's Phœbe.

N. E. III.

Western species, only once recorded from New England; casual in Illinois and Wisconsin.

CONTOPUS BOREALIS (SWAINS.).

Olive-sided Flycatcher.

N. E. Ill.

CONTOPUS VIRENS (LINN.).

Wood Pewee. Flycatcher. N. E. Fla. Ill.

352 (463) EMPIDONAX FLAVIVENTRIS BAIRD.

Yellow-bellied Flycatcher. N. E. Fla. Ill.

353 (465) EMPIDONAX VIRESCENS (VIEILL.).

Acadian Flycatcher.

N. E. Fha. III.

EMPIDONAX TRAILLII ALNORUM BRETSTER.

Alder Flycatcher. N. E. 111.

355 (467) EMPIDONAX MINIMUS BAIRD.

Least Flycatcher.

N. E. Fla. 111. 
FAMILY ALANDIDA. Larks. A. o. U.

356 (473) ALAUDA ARVENSIS LINN.

European Skylark.

Accidental in Greenland.

357 (474) OTOCORIS ALPESTRIS (LINN.).

Horned Lark.

Shore Lark.

N. E. M1.

358 (4746) OTOCORIS ALPESTRIS PRATICOLA HENSH.

Prairie Horned Lark.

N. E. II1.

FAIILY CORVIDAE. Crows, Jays, and Magpies.

359 (475) PICA PICA HUDSONICA (SAB.).

American Magpie.

A western species; stragglers have been recorded from Illinois and Montreal, Canada.

$L$

360 (477) CYANOCITTA CRISTATA (LINN.).

Blue Jay.

N. E. Ill.

361 (477a) CYANOCITTA CRISTATA FLORINCOLA COUES.

Florida Blue Jay.

Fia.

362 (479) APHELOCOMA FLORIDANA (BARTR.).

Florida Jay.

Fla.

363 (484) PERISOREUS CANADENSIS (LINN.).

Canada Jay.

N. E.

364 (484) PERISOREUS CANADENSIS NIGRICAPILLUS RIDGW. Labrador Jay.

365 (486a) CORVUS CORAX PRINCIPALIS RIDGW.

Northern Raven.

N. E. Ill.

1 - 366 (488) CORVUS AMERICANUS AUD.

American Crow.

N. E. Fla. IIl.

367 (488 $q$ ) CORVUS AMERICANUS FLORIDANUS BAIRD.

Florida Crow.

Fla.

368 (490) CORVUS OSSIFRAGUS WILS.

Fish Crow.

N. E. Fla.

FAMILY STURNIDE. Starlings.

369 (493) STURNUS VULGARIS IINN.

Starling.

An old world species, accidental in Greenland. Some years ago it was introduced iu Now York, and is now not uncommon in

Central Park. 
FAMILY ICTERIDAE. Blackbirds, Orioles, etc. A. .0. . .

370 (494) DOLICHONYX ORYZIVORUS (LINN.).

Bobolink.

N. E. Fla. I11.

$L^{2} 371$ (195) MOLOTHRUS ATER (BODD.).

Cowbird.

N. F. Fli.. Ill.

2 372 (497) XANTHOCEPHALUS XANTHOCEPHALUS (BONAP.).

Yellow-headed Blackbird.

N. F. Fla. Ill.

L 373 (498) AGELAIUS PHENICEUS (LINA.).

Red-winged Blackbird.

N. E. Fla. Ill.

374 (4986) AGELAIUS PHCNICEUS BRYANTI RIDGW.

Bahaman Red-winged Blackbird.

Fla.

L 375 (501) STURNELLA MAGNA (LIXY.).

Meadowlark.

N. E. Fla. $\Pi 1$.

L 376 (5010) STURNELLA MAGNA NEGLECTA (AUD.).

Western Mcadowlark.

Ill.

377 (502) ICTERUS ICTERUS (LINT.).

Troupial.

South American species, recorded by Audubon from South Carolina; no subsequent records for eastern North America.

L 378 (506) ICTERUS SPURIUS (LINX.).

Orchard Oriole.

N. E. Fla. Ill.

L 379 (507) ICTERUS GALBULA (LMX.).

Baltimore Oriole.

N. E. Fla. Ill.

(3) (.1) ICTERUS BULLOCKI (SWAIXS.).

Bullock's Oriole.

Western species, only once recorded from our Eastern States; Bangor, Me., one record.

L-381 (509)

SCOLECOPHAGUS CAROLINUS (MÜLL.).

Rusty Blackbird.

N. E. Ill.

SCOLECOPHAGUS CYANOCEPHALUS (WAGL.).

Brewer's Blackbird.

IIl.

A western species, which sometimes occurs in Illinois; stragglers have been killed in South Carolina and Louisiana.

383 (511) QUISCALUS QUISCULA (IINN.).

Purple Grackle.

N. E.

- 384 (511a) QUISCALUS QUISCULA AGLEUS (I3.1MRD).

Florida Grackle.

Fla. 
A. o. U,
No.

385 (5116) QUISCALUS QUISCULA RENEUS (RIDGW.).

Bronzed Grackle. N. E. Ill.

386 (513) QUISCALUS MAJOR (VIEILL.).

Boat-tailed Grackle.

Fla.

FAIMILY FRINGILLID E. Finches, Sparrows, etc.

387 (514) COCCOTHRAUSTES VESPERTINA (COOP.).

Evening Grosbeak.

N. E. 111 .

1 - 388 (515) PINICOLA ENUCLEATOR (LINN.).

Pine Grosbeak.

N. E. Ill.

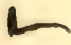

389 (517) CARPODACUS PURPUREUS (GMEL.).

Purple Finch.

N. E. Fla. III.

390 PASSER DOMESTICUS (LINN.).

English Sparrow.

N. E. Fla. Ill.

European species, introduced in United States.

391 PASSER MONTANUS.

European Tree Sparrow.

N. E.?

European species, iutroduced in United States.

392 (521) LOXIA CURVIROSTRA MINOR (BREHM).

American Crossbill.

N. E. I11.

393 (522) LOXIA LEUCOPTERA GMEL.

White-winged Crossbill.

N. E. Ill.

394 (527) ACANTHIS HORNEMANNII (HOLB.).

Greenland Redpoll.

$395(52 \pi)$ ACANTHIS HORNEMANNII EXILIPES (COUES).

Hoary Redpoll.

N. E. III.

(i.: (5:*) ACANTHIS LINARIA (LINN.).

Redpoll. Redpoll Linnet.

N. E. Ill.

397 (528 $(\imath)$ ACANTHIS LINARIA HOLBCELLII (BREIM).

Holbœll's Redpoll.

N. E.

398 (5287,) ACANTHIS LINARIA ROSTRATA (COUES).

Greater Redpoll.

N. E. IIl.

399 CARDUELIS CARDUELIS TINN.

European Goldfinch.

N. E.

Tntroduced in Massachusetts and New York.

400 (529) SPINUS TRISTIS (LINN.).

American Goldfinch. Yellow Bird. N. E. Fla. Ill. 
A. o. U.
sii.

401 (532) SPINUS NOTATUS (DUBUS).

Black-headed Goldfinch.

Mexican species, recorded by Audubon from Kentucky.

402 (5.33) SPINUS PINUS (WIIS.).

Pine Siskin. Pine Finch. N.'E. Fla. Ill.

403 (531) PLECTROPHENAX NIVALIS (LINN.).

Snowflake.

Snow Bunting.

N. E. Fla. III.

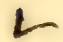

404 (536) CALCARIUS LAPPONICUS (LINN.).

Lapland Longspur.

N. E. I11.

L $405(537)$ CALCARIUS PICTUS (SWAINS.).

Smith's Longspur.

I11.

406 (538) CALCARIUS ORNATUS ('IOWNS.).

Chestnut-collared Longspur.

N. E.

Western species, accidental in Massachusetts and New York.

$\sim 407(539)$

RHYNCHOPHANES MCCOWNII (LAWR.).

McCown's Longspur.

III.

Western species, casual in Illinois.

$405(540)$

POOCETES GRAMINEUS (GMEL.).

Vesper Sparrow.

Bay-winged Bunting. N. E. Fla. Ill.

409 (541) AMMODRAMUS PRINCEPS (MATN.).

Ipswich Sparrow.

N. E.

L 410 (542 $a$ ) AMMODRAMUS SANDWICHENSIS SAVANNA (WILS.).

Savanna Sparrow. N. L. Fla. Ill.

4411 (516) AMMODRAMUS SAVANNARUM PASSERINUS (WILS.).

Grasshopper Sparrow.

Yellow-winged Sparrow.

N. E. Fla. Ill.

L 412 (547) AMMODRAMUS HENSLOWII (AUD.).

Henslow's Sparrow. N. E. Fla. Ill.

$L 413$ (548) AMMODRAMUS LECONTEII (AUD。).

Leconte's Sparrow.

FIa. Ill.

414. (549) AMMODRAMUS CAUDACUTUS (GMET.).

Sharp-tailed Sparrow. Sharp-tailed Finch. N. E. Fln.

L 415 (5.19) AMMODRAMUS CAUDACUTUS NELSONI ALLIN.

Nelson's Sparrow.

N. E. 111 .

416 (5496) AMMODRAMUS CAUDACUTUS SUBVIRGATUS

Acadian Sharp-tailed Sparrow.

DIVIGITT.

N. E. 
A. O. T.

417 (550) AMMODRAMUS MARITIMUS (WILS.).

Seaside Sparrow.

Seaside Finch.

N. E. Fla.

$118(550 a)$ AMMODRAMUS MARITIMUS PENINSULA ALLEN.

Scott's Seaside Sparrow.

Fla.

419 (551) AMMODRAMUS NIGRESCENS RIDGW.

Dusky Seaside Sparrow.

Fla.

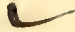

$420(55:)$

CHONDESTES GRAMMACUS (SAT).

Lark Sparrow. Lark Finch.

N. E. Fla. Ill.

4 421 (553) ZONOTRICHIA QUERULA (NUTT.).

Harris's Sparrow.

I11. (rare).

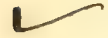

$422(554)$

ZONOTRICHIA LEUCOPHRYS (FORST.).

White-crowned Sparrow.

N. E. III.

L

423 (558) ZONOTRICHIA ALBICOLLIS (GMEL.).

White-throated Sparrow.

N. E. Fla. Ill.

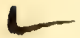

424 (559) SPIZELLA MONTICOLA (GMEL.).

Tree Sparrow.

N. E. Ill.

L 425 (560) SPIZELLA SOCIALIS (IVILS.).

Chipping Sparrow.

N. E. Fla. Ill.

12426 (561) SPIZELLA PALLIDA (SWAINS.).

Clay-colored Sparrow.

I11.

427 (562) SPIZELLA BREWERI CASS.

Brewer's Sparrow.

N. H.

Western species, once taken in Massachusetts.

$1 \quad 128$ (563) SPIZELLA PUSILLA (WILS.).

Field Sparrow.

N. E. Fla. Ill.

429 (563a) SPIZELLA PUSILLA ARENACEA CHAdB.

Western Field Sparrow.

Accidental in southern Louisiana.

ᄂ 430 (567) JUNCO HYEMALIS (LINN.).

Slate-colored Junco. Snowbird. N. E. Fla. 111.

431 (567a) JUNCO HYEMALIS OREGONUS (TOWNS.).

Oregon Junco.

Western species, recordod from Illinois; one record.

432 JUNCO HYEMALIS SHUFELDTI COALE.

Shufeldt's Junco.

N. E. I11.

Western species, accidental in Massachusetts, Illinois, and Maryland.

leare the

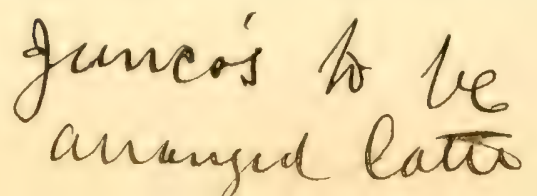




$$
\begin{aligned}
& \text { A. o. } \mathbb{~} \text {. } \\
& \text { No. }
\end{aligned}
$$

133 (567.1) JUNCO HYEMALIS CAROLINENSIS BRETST,

Carolina Junco.

Alleghany Mountains, from Virginia to the Carolinas.

434 (575) PEUCAEA RSTIVALIS (LICIT.).

Pine-woods Sparrow.

Fla.

L 435 (5รวa) PEUCAEA AESTIVALIS BACHMANII (AUD.).

Bachman's Sparrow.

Fla. III.

436 (581) MELOSPIZA FASCIATA (GMEL.).

Song Sparrow.

N. E. Flit. Ill.

4437 (5S3) MELOSPIZA LiNCOLNI (AUD.).

Lincoln's Sparrow.

N. E. 111 .

135 (5S4) MELOSPIZA GEORGIANA (LATH.).

Swamp Sparrow.

N. E. Fla. IIl.

2439 (5ังรั) PASSERELLA ILIACA (MERR.).

Fox Sparrow. N. E. Fla. Ill.

4410 (587) PIPILO ERYTHROPHTHALMUS (LIN.).

Towhee.

N. E. Fla. III.

411 (587a) PIPILO ERYTHROPHTHALMUS ALLENI COLES.

White-eyed Towhee.

Fla.

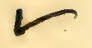

412 (593) CARDINALIS CARDINALIS (LINN.).

Cardinal. Cardinal Grosbeak.

N. E. Flix. IIl.

413 (595) HABIA LUDOVICIANA (LiNN.).

Rose-breasted Grosbeak.

N. E. Fla, III.

444 (596) Habia MELANOCEPHalA (Swains.).

Blackheaded Grosbeak.

A western species, which has been taken in Michigan.

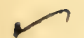

415 (597) GUIRACA CAERULEA (LINN.).

Blue Grosbeak.

N. E. Fla. Ill.

416 (59S) PASSERINA CYANEA (LINN.).

Indigo Bunting.

N. E. Fla. Ill.

147 (600) PASSERINA VERSICOLOR (BONAP.).

Varied Bunting.

Western species, which has been taken in Michigan.

C 4t (601) PASSERINA CIRIS (LINN.).

Painted Bunting.

Fla. 111.

449 (603) EUETHEIA BICOLOR (LINN.).

Grassquit. Black-faced Finch.

West Indian species, recorded from Florida Keys. 
A. o. U.
No.

450 (603.1) EUETHEIA CANORA (GMEL.).

Melodious Grassquit.

Cuban Finch.

Cuban species, recorded from Florida Keys.

451 (604) SPIZA AMERICANA (GMEL.).

Dickcissel.

Black-throated Bunting.

N. E. I11.

452 (605) CALAMOSPIZA MELANOCORYS STEJN.

Lark Bunting.

Western species, recorded from Massachusetts and Long Island.

FAMILY TANAGRID $\notin$. Tanagers.

453 (607) PIRANGA LUDOVICIANA (WILS.).

Louisiana Tanager.

N. E.

Western species, recorded from Massachusetts and New York.

$\checkmark$

454 (608) PIRANGA ERYTHROMELAS VIEILI.

Scarlet Tanager.

N. E. Fla. Ill.

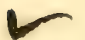

455 (610) PIRANGA RUBRA (LINN.).

Summer Tanager.

N. E. Fla. Ill.

FAMILY HIRUNDINIDA. Swallows.

456 (611) PROGNE SUBIS (IANN.).

Purple Martin.

N. E. Fla. Ill.

457 (611.1) PROGNE CRYPTOLEUCA BAIRD.

Cuban Martin.

Fla.

L 458 (612) PETROCHELIDON LUNIFRONS (SAT).

Cliff Swallow.

N. E. 111.

459

PETROCHELIDON FULVA (VIEILL.).

Cuban Cliff Swallow.

West Indian species, accidental at Dry Tortugas, Fla.

460

CALLICHELIDON CYANEOVIRIDIS (BRYANT).

Bahaman Swallow.

Bahaman species, accidental at Dry Tortugas, Fla.

461 (613) CHELIDON ERYTHROGASTER (BODD.).

Barn Swallow.

N. F. 111.

C 462 (614) TACHYCINETA BICOLOR (VIEIM...).

Tree Swallow.

White-bellied Swallow.

N. E. Fla. III. 
2 A. No.

463 (616) CLIVICOLA RIPARIA (LINN.).

Bank Swallow.

STELGIDOPTERYX SERRIPENNIS (AUD.).

Rough-winged Swallow.

Y. E. Fla. Ill.

FAMILY AMPELIDAE. Waxwings.

465 (618) AMPELIS GARRULUS LINN.

Bohemian Waxwing.

N. E. Ill.

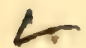

466 (619) AMPELIS CEDRORUM (VIEILL.).

Cedar Waxwing.

N. E. Fla. Ill.

FANILY LANIIDA. Shrikes.

2467 (621) LANIUS BOREALIS VIEILL.

Northern Shrike.

Butcher Bird.

468 ( (j2:) LANIUS LUDOVICIANUS LINN.

Loggerhead Shrike.

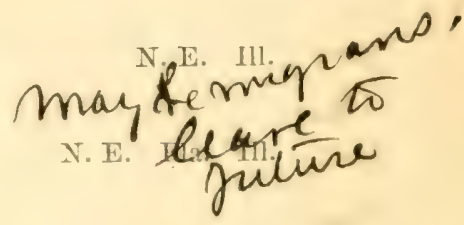

FAMILY VIREONIDA. Vireos.

469 (623) VIREO CALIDRIS BARBATULUS (CAB.).

Black-whiskered Vireo.

Fla.

L 470 (624) VIREO OLIVACEUS (IINN.).

Red-eyed Vireo.

N. E. Fla. Ill.

471 (625) VIREO FLAVOVIRIDIS (CASS.).

Yellow-green Vireo.

Mexican species, once recorded from Province of Quebec.

1 47.) (626) VIREO PHILADELPHICUS (CASS.).

Philadelphia Vireo.

N. E. Fla. 111.

1 473 (627) VIREO GILVUS (VIEILL.).

Warbling Vireo.

N. F. Fla. Ill.

L 4.4 (628) VIREO FLAVIFRONS VIEILL.

Yellow-throated Vireo.

N. E. Flit.

VIREO SOLITARIUS (IVILS.).

Blue-headed Vireo.

N. E. Fla.

$47($ (6296) VIREO SOLITARIUS PLUMBEUS (COUES).

Plumbeous Vireo.

Western species, one record in New York State. 
A. $0 . \mathrm{U}$.
No.

477 (629c) VIREO SOLITARIUS ALTICOLA BREWST.

Mountain Solitary Vireo.

Fla.

178 (6:31) VIREO NOVEBORACENSIS (GMEL.).

White-eyed Vireo.

N. E. Flat. Ill.

47: (631 $a$ ) VIREO NOVEBORACENSIS MAYNARDI BREWST.

Key West Vireo.

Southern Fla.

4 S9 (633) VIREO BELLII (AUd.).

Bell's Vireo.

III.

FAMILY CEREBID峟. Honey Creepers.

481 (635) CCEREBA BAHAMENSIS (REICI.).

Bahama Honey Creeper.

Bahaman species; stragglers have been taken on the Florida Keys.

FAMILY MNIOTILTIDA. Wood Warblers.

482 (636) MNIOTILTA VARIA (LINN.).

Black and White Warbler.

N. E. Fla. Ill.

1 483 (637) PROTONOTARIA CITREA (BODD.).

Prothonotary Warbler.

N. E. Fla. Ill.

ᄂ 484 (638) HELINAIA SWAinsoniI Aud.

Swainson's Warbler.

Fla. III.

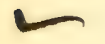

485 (639) HELMITHERUS VERMIVORUS (GMEL.).

Worm-eating Warbler.

N. E. Fla. IIl.

486 (640) HELMINTHOPHILA BACHMANI (AUD.).

Bachman's Warbler.

Fla.

L 487 (641) HELMINTHOPHILA PINUS (LINN.).

Blue-winged Warbler.

N. E. Fla. IIl.

ᄂ

488 (642) HELMINTHOPHILA CHRYSOPTERA (LINN.).

Golden-winged Warbler.

N. E. Fla. Ill.

489

HELMINTHOPHILA LAWRENCEI (HERRICK).

Lawrence's Warbler.

N. E.

Probably a hybrid between H. pinus and $\mathbf{H}$. chrysoptera.

490

HELMINTHOPHILA LEUCOBRONCHIALIS (BREWST.).

Brewster's Warbler.

N. E.

Probably a hybrid between $\mathrm{H}$. pinus and $\mathrm{H}$. chrysoptera.

491 (645) HELMINTHOPHILA RUFICAPILLA (WILS.).

Nashville Warbler.

N. E. Fla. Ill. 
1 A. N.U.

HELMINTHOPHILA CELATA (SAY).

Orange-crowned Warbler.

N. E. Fla. Ill.

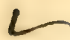

493 (6.4) HELMINTHOPHILA PEREGRINA (IVILS.)

Tennessee Warbler.

N. E. Fla. 111.

494 (0.8) COMPSOTHLYPIS AMERICANA (INY.'

Parula Warbler.

N. E. Flis. Ill.

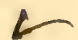

495 (650) DENDROICA TIGRINA (GIEL.).

Cape May Warbler.

N. E. Fla. III

4796 (652) DENDROICA RESTIVA (GMEL.).

Yellow Warbler.

N. E. Fla. Ill.

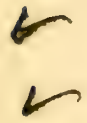

$497(654)$

DENDROICA CERULESCENS (GMEL.).

Black-throated Blue Warbler.

N. E. Fla. 111.

$498,655)$

DENDROICA CORONATA (LINN.).

N. E. Fla. Ill.

Yellow-rumped Warbler.

Myrtle Warbler.

N. E. Fla. Ill.

499 (656) DENDROICA AUDUBONI ('TOWNS.).

Audubon's Warbler.

N. E.

A western species; stragglers have been taken in Massachusetts and Pennsylvania.

L $500(657)$

DENDROICA MACULOSA (GMEL.).

Magnolia Warbler.

Black and Yellow Warbler.

N. E. Fla. Ill.

L 501 (65S) DENDROICA CAERUEA (TILS.).

Cerulean Warbler.

N. E. Fla. Ill.

ᄂ 502 (659) DENDROICA PENNSYLVANICA (LINN.),

Chestnut-sided Warbler.

N. E. Fla. III.

L. 503 (660) DENDROICA CASTANEA (WILS.).

Bay-breasted Warbler.

N. E. Fla. Ill.

L 504 (661) DENDROICA STRIATA (Forst.).

Black-poll Warbler.

N. T. Fla. 111.

ᄃ 505 (662) DENDROICA BLACKBURNIA (GMEL.).

Blackburnian Warbler.

N. E. Fla. Ill.

506 (663) DENDROICA DOMINICA (LINN.).

Yellow-throated Warbler.

N. E. Fla.

L 507 (663a) DENDROICA DOMINICA ALBILORA 13AMID.

Sycamore Warbler.

III.

$150 S$ (667) DENDROICA VIRENS (GMEL.).

Black-throated Green Warbler.

N. E. Fla. Ill. 


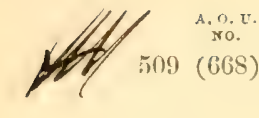

DENDROICA TOWNSENDI (NUTT.).

Townsend's Warbler.

A western species; one record from Pennsylvania.

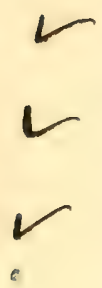

510 (ito) DENDROICA KIRTLANDI BAIRD.

Kirtland's Warbler.

Fla. IIl.

511 (iit) DENDDROICA VIGORSII (AUn.).

Pine Warbler.

N. E. Fla. III.

51.2 (672) DENDROICA PALMARUM (GMEL.).

Palm Warbler.

Red Poll Warbler.

N. F. Fla. Ill.

51: (672a) DENDROICA PALMARUM HYPOCHRYSEA IIIDGW.

Yellow Palm Warbler.

N. E. Fli

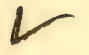

$51+(673)$

DENDROICA DISCOLOR (VIEILL.).

Prairie Warbler.

N. E. Fla. Ill.

515 (674) SEIURUS AUROCAPILLUS (IINN.).

Oven-bird.

N. E. Fla. Ill.

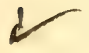

$516(675)$

SEIURUS NOVEBORACENSIS (GMLL.).

Water-Thrush.

N. E. Fla. Ill.

$\angle 517(675 a)$ SEIURUS NOVEBORACENSIS NOTABILIS (GRINN.).

Grinnell's Water-Thrush.

III.

Illinois, westward; occasionally east to Virginia and New Jersey

2518 (676) SEIURUS MOTACILLA (VIEILL.).

Louisiana Water-Thrush.

N. E. Fla: Ill.

C 519 (677) GEOTHLYPIS FORMOSA (WILS.).

Kentucky Warbler.

N. E. Fla. Ill.

$520(678)$ GEOTHLYPIS AGILIS (WILS.).

Connecticut Warbler.

N. E. Fla. 111 .

521 (679) GEOTHLYPIS PHILADELPHIA (IVIL.).

Mourning Warbler.

N. E. 111 .

522 (6SI) GEOTHLYPIS TRICHAS (IINN.).

Maryland Yellow-throat.

N. E. Flı. IH.

523 GEOTHLYPIS TRICHAS IGNOTA CIIAMAN.

Florida Yellow-throat.

Jlat.

L 521 (683) ICTERIA VIRENS (IINN.).

Yellow-breasted Chat.

‥ F. 111.

$L$ 52: (G81) SYLVANIA Mitrata (GMEL.).

Hooded Warbler.

N. W. Fla, III. 


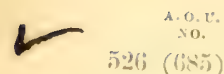

SYLVANIA PUSILLA (WILS.).

Wilson's Warbler.

N. I:. III.

1 527 (686) sylvania Canadensis (IINn.).

Canadian Warbler.

N. E. III.

528 (687) SETOPHAGA RUTICILLA (LiNy.).

American Redstart.

N. F. Fla. 111 .

FAMILY MOTACILLIDAE. Wagtails.

529 (69) MOTACILLA ALBA LiNN.

White Wagtail.

European species, accidental in Greenland.

L 530 (697) ANTHUS PENSILVANICUS (LATi.).

Titlark. American Pipit.

N. E. Fla. Ill.

531 (698) ANTHUS PRATENSIS (LINN.).

Meadow Pipit.

European species, accidental in Greenland.

53:2 (700) ANTHUS SPRAGUEII (AUD.).

Sprague's Pipit.

Westem species, Charleston, S. C., one record.

FAIIIY TROGLODYTIDE. Wrens, Thrashers, etc. L 533 (703) MIMUS POLYGLOTTOS (LINN.).

Mockingbird. N. E. Fla. Ill.

3

534 (704) GALEOSCOPTES CAROLINENSIS (LINN.).

Catbird.

N. E. Fla. Ill.

L. 535 (705) HARPORHYNCHUS RUFUS (LINN.).

Brown Thrasher.

Brown Thrush. N. E. Fla. Ill.

L 536 (718) THRYOTHORUS LUDOVIĊIANUS (LATI.).

Carolina Wren. $N$ N. E. Fla. III.

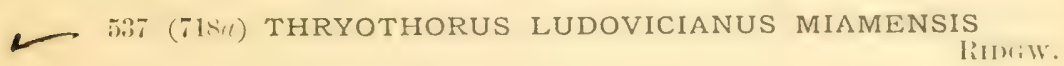

Florida Wren. Fla.

L 538 (719) THRYOTHORUS BEWICKII (AUD.).
Bewick's Wren. 
A. 0 V.

$\mathcal{L} 539(721)$

TROGLODYTES AËDON VIEILL.

House Wren.

N. E. Fla. III.

2540 (7216) TROGLODYTES AËDON AZTECUS (BATRD).

Western House Wren.

$\longleftarrow$

541 (722) TROGLODYTES HIEMALIS VIEILL.

Winter Wren.

N. E. Fla.

742 (724) CISTOTHORUS STELLARIS (LICHT.).

Short-billed Marsh Wren.

N. T. Fla. Ill.

$\longleftarrow$

$543(725)$

CISTOTHORUS PALUSTRIS (WILS.).

Long-billed Marsh Wren.

N. E. Fla. Ill.

.4. CISTOTHORUS PALUSTRIS GRISEUS BREWST.

Worthington's Marsh Wren.

545 (725.1) CISTOTHORUS MARIANE SCOTT.

Marian's Marsh Wren.

Fla.

FAMILY CERTHIIDAE. Creepers.

$\tau_{546(726)}$

CERTHIA FAMILIARIS AMERICANA (BONAP.).

Brown Creeper.

N. E. Fla. IIl.

FAMILY PARID $Æ . \quad$ Nuthatches and Tits.

L 517 (727) SITTA CAROLINENSIS IATHI.

White-breasted Nuthatch.

X. E. IH.

itu (727a) SITTA CAROLINENSIS ATKINSI (SCOTT).

Florida White-breasted Nuthatch.

Fla.

$\checkmark$ il!) (728) SITTA CANADENSIS LINN.

Red-breasted Nuthatch.

N. E. Ill.

i.j) (72!) SitTa PUSilla Iatit.

Brown-headed Nuthatch.

Fla.

L 5 il (731) PARUS BICOLOR (LINN.).

Tufted Titmouse.

N. E. Fla. III.

5.12 (735) PARUS ATRICAPILLUS (LINN.).

Chickadee.

N. E. III.

75i3 (736) PARUS CAROLINENSIS ORD.

Carolina Chickadee.

Fla. III.

551 (740) PARUS HUDSONICUS Forst.

Hudsonian Chickadee.

N. E. IIl. 
FAMILY SYLVIID E. Warblers, Kinglets, Gnatcatchers.
A. o. v.
No.
555 (748) REgULUS SATRAPA Licht.
Golden-crowned Kinglet.
N. E. Fla. Ill.
556 (749) REgulus CALENDUla (LiNN.).
Ruby-crowned Kinglet.
N. E. Fla. Ill.
POLIOPTILA CAERULEA (LiNN.).
Blue-gray Gnatcatcher.
N. E. Fla. IIl.
FAMILY TURDIDAE. Thrushes, Solitaires, Stone- chats, Bluebirds, etc.

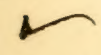
558 (754) MYADESTES TOWNSENDII (AUD.).
Townsend's Solitaire.
Western species, accidental in rllinois; one record.

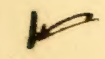
$559(755)$
TURDUS MUSTELINUS GMEL.
Wood Thrush.
N. E. Fla.
L
$560(756)$
TURDUS FUSCESCENS STEPH.
Wilson's Thrush.
N. E. Fla.
- 561 (756a) TURDUS FUSCESCENS SALICICOLUS (RIDGW.).
Willow Thrush.
III.
L- 562 (757) TURDUS ALICIE BAIRD.
Gray-cheeked Thrush. N. E. Fla. Ill.
L 563 (757a) TURDUS ALICI E BICKNELLI (RIDGW.).
Bicknell's Thrush.
N. E. III.
L_ $564(758 a)$ TURDUS USTULATUS SWAINSONII (CAB.).
Olive-backed Thrush. N. E. Fla. Ill.
1565 (7596) TURDUS AONALASCHKE PALLASII (CAB.).
Hermit Thrush. N. E. Fla. Ill.
566 (760) TURDUS ILIACUS LINN.
Red-winged Thrush.
European species, accidental in Greenland.
2 - 567 (761) MERULA MIGRATORIA (LINN.).
American Robin.
N. E. Fla. Ill. 
$4^{2}$

568 (763) HESPEROCICHLA NÆVIA (GMEL.).

Varied Thrush.

N. E. Western species; stragglers have been taken in Massachusetts,
Long Island, and New Jersey.

569 (765) SAXICOLA CENANTHE (LINN.). Wheatear.

Old-world species, which occur in Greenland and Labrador; stagoglers have been taken in Nova Scotia, Maine, and Long
Island.

570 (766) SIALIA SIALIS (LiNN.).

Bluebird.

N. E. Fla. 111.

Scalia arcticia do nits steele on line untie? ae canangumentr.

66531 

